\title{
OPTIMIZATION OF THE FIRST STEKLOV EIGENVALUE IN DOMAINS WITH HOLES: A SHAPE DERIVATIVE APPROACH
}

\author{
JULIÁN FERNÁNDEZ BONDER, PABLO GROISMAN AND JULIO D. ROSSI
}

\begin{abstract}
The best Sobloev trace constant is given by the first eigenvalue of a Steklov-like problem. We deal with minimizers of the Rayleigh quotient $\|u\|_{H^{1}(\Omega)}^{2} /\|u\|_{L^{2}(\partial \Omega)}^{2}$ for functions that vanish in a subset $A \subset \Omega$, which we call the hole. We look for holes that minimize the best Sobolev trace constant among subsets of $\Omega$ with prescribed volume. First, we find a formula for the first variation of the first eigenvalue with respect to the hole. As a consequence of this formula, we prove that when $\Omega$ is a ball the symmetric hole (a centered ball) is critical when we consider deformations that preserves volume but is not optimal. Finally, we prove that by the Finite Element Method we can approximate the optimal configuration and, by means of the shape derivative, we design and algorithm to compute the discrete optimal holes.
\end{abstract}

\section{InTRODUCTION.}

Sobolev inequalities are relevant for the study of boundary value problems for differential operators. They have been studied by many authors and is by now a classical subject. It at least goes back to [1], for more references see [5]. In particular, the Sobolev trace inequality has been intensively studied in $[2,6,7,8$, $9,10,16,17,18]$, etc.

Let $\Omega \subset \mathbb{R}^{N}$ be a bounded smooth domain $\left(C^{2}\right.$ is enough for our arguments). For any subset $A \subset \bar{\Omega}$, which is a smooth open subset (also $C^{2}$ ) we define $H_{A}^{1}(\Omega)=$ $\left\{u \in H^{1}(\Omega):\left.u\right|_{A} \equiv 0\right\}$. We refer to $A$ as the hole. There is a compact embedding (known as the Sobolev trace theorem) $H_{A}^{1}(\Omega) \hookrightarrow L^{2}(\partial \Omega)$. Hence there exists a constant $S=S(A)$ such that, $S\|u\|_{L^{2}(\partial \Omega)}^{2} \leq\|u\|_{H^{1}(\Omega)}^{2}$, for all $u \in H_{A}^{1}(\Omega)$. The best Sobolev trace constant is the largest $S$ such that the above inequality holds, that is,

$$
S=S(A)=\inf _{v \in H_{A}^{1}(\Omega) \backslash H_{0}^{1}(\Omega)} \frac{\int_{\Omega}|\nabla v|^{2}+v^{2} d x}{\int_{\partial \Omega} v^{2} d S} .
$$

Since the embedding is compact, we have existence of extremals, i.e. functions where the infimum is attained. These extremals are strictly positive in $\Omega \backslash \bar{A}$, smooth up to the boundary and are weak solutions of the following Steklov-type

Key words and phrases. Steklov eigenvalues, Sobolev trace embedding, shape derivative. 2000 Mathematics Subject Classification. 35P15, 49K20, 49M25, 49Q10.

Supported by Fundacion Antorchas, CONICET and ANPCyT PICT No. 03-05009. JFB is also supported by ANPCyT PICT No. 03-10608. 
eigenvalue problem:

$$
\begin{cases}-\Delta u+u=0 & \text { in } \Omega \backslash \bar{A} \\ \frac{\partial u}{\partial \nu}=\lambda u & \text { on } \partial \Omega, \\ u=0 & \text { on } \partial A,\end{cases}
$$

where $\partial / \partial \nu$ is the unit normal exterior derivative. The first eigenvalue of (1.1) coincides with the best Sobolev trace constant, $S(A)=\lambda_{1}(A)$.

We normalize the extremals, or eigenfunctions, with

$$
\int_{\partial \Omega} u^{2} d S=1
$$

This eigenvalue problem has been introduced by [22] and has been intensively investigated ever since. See, for instance, $[3,11,18]$.

Optimization problems for eigenvalues have been extensively studied in the literature due to the many applications in several branches of applied mathematics and engineering, specially in optimal design problems, see the survey [13].

Optimal design problems are usually formulated as problems of minimization of the energy stored in the design under a prescribed loading. For applications to engineering of optimization for Steklov eigenvalues, see [3].

In view of the above discussion, we consider the following optimization problem:

For a fixed $0<\alpha<|\Omega|$, find a set $A^{*}$ of measure $\alpha$ that minimizes $\lambda_{1}(A)$ among all measurable subsets $A \subset \Omega$ of measure $\alpha$.

This problem was first studied in [11] where the authors show that such an optimal set exists among the class of measurable subsets of $\Omega$.

The topology of this optimal set is not known but the complement, $\Omega \backslash A$, is connected (see [11]).

Some symmetry is expected in the case that $\Omega$ is a ball. It is shown in [11] that every optimal set is spherically symmetric in the sense of [21].

On the other hand, it is also proved in [11] that a set $A$ that maximizes $\lambda_{1}$ does not exist. In fact, $\sup \lambda_{1}(A)=+\infty$ where the supremum is taken over all sets $A$ of given measure $\alpha$.

In a subsequent paper, [12], the interior regularity of this optimal hole $A^{*}$ was studied. In that paper it is proved, under very mild assumptions on the eigenfunction that are satisfied, for instance, if $\Omega$ is a ball, that the optimal set $A^{*}$ is an open set with smooth boundary.

The purpose of this article is twofold. First we analyze the dependence of the first Steklov eigenvalue with respect to the hole by means of the so called shape derivative. We compute the derivative of $\lambda_{1}(A)$ with respect to variations of the hole and use this formula to deduce some symmetry (or lack of symmetry) properties of optimal holes, namely, if $\Omega$ is a ball then the centered ball is not optimal, but it is critical with respect to deformations that preserve volume.

Second, we turn our attention to numerical approximations of optimal holes. We believe that these are our main results. In this direction we first prove that if we consider a finite element discretization of the problem, then the discrete optimal hole 
converges measure theoretically to the continuous one. Then we face the problem of computing the discrete optimal hole. We design an algorithm based in the shape derivative of the first Steklov eigenvalue. This algorithm is described in Section 3.2 and some numerical experiments are given in Section 3.3. This approach to numerical approximation in optimal design problems has been extensively used in engineering, in quite a lot of situations (building, car, aircraft, aerospace industries, etc.), see for example [19].

1.1. Description of the results. The main results of the paper are stated below.

To study the variations of the first Steklov eigenvalue with respect to variations of the domain we consider a smooth map ( $C^{1}$ is enough) $V: \mathbb{R}^{N} \rightarrow \mathbb{R}^{N}$, the deformation field. Since we are interested in variations of the hole we will assume that $\operatorname{supp}(V) \subset \Omega$. Hence, given a closed set $A$, we consider the perturbed holes,

$$
A_{t}:=(I d+t V)(A)=\{x+t V(x), x \in A\} .
$$

Our first result is the following:

Theorem 1. The function $\lambda_{1}\left(A_{t}\right)$ is differentiable with respect to $t$ at $t=0$ and it holds

$$
\left.\frac{d}{d t} \lambda_{1}\left(A_{t}\right)\right|_{t=0}=\lambda_{1}^{\prime}(A)=-\int_{\partial A}\left(\frac{\partial u}{\partial \nu}\right)^{2}\langle V, \nu\rangle d S,
$$

where $u$ is a normalized eigenfunction and $\nu$ is the exterior normal vector to $\Omega \backslash \bar{A}$.

As a consequence of the above theorem and the regularity of optimal holes proved in [12] we get a necessary condition on the holes to be optimal.

Corollary 1.4. Let $A^{*}$ be a smooth optimal hole (i.e. $\lambda_{1}\left(A^{*}\right)=\min _{|A|=\alpha} \lambda_{1}(A)$ ) and $u$ an eigenfunction associated to $\lambda_{1}\left(A^{*}\right)$, then $\frac{\partial u}{\partial \nu}$ is constant along $\partial A^{*}$.

This condition was already observed in [12].

Next, we look for the optimal hole when $\Omega$ is a ball $B(0, R)$. As a consequence of Theorem 1, we have that a centered ball $A=B(0, r), r<R$ is critical in the sense that $\lambda_{1}^{\prime}(A)=0$ when considering deformations that preserve volume, that is, deformations that satisfy $\operatorname{div}(V)=0$ in $A$. Surprisingly, this configuration is not optimal, thus we are in presence of a lack of symmetry in the optimal configuration. To prove this fact we use ideas from [16]. When the measure of the hole is half the measure of the ball, we show that half the ball is not optimal either.

Theorem 2. Let $\Omega=B(0, R)$.

(1) Let the hole be a centered ball $A=B(0, r)$. Then,

(a) this configuration is critical in the sense that $\lambda_{1}^{\prime}(A)=0$ for all deformations $V$ that preserve the volume of $A$.

(b) This symmetric configuration is not optimal.

(2) The half ball $B^{+}(0, R):=B(0, R) \cap\left\{x_{1} \geq 0\right\}$ is not optimal.

Now we turn our attention to the problem of computing the optimal holes. First, we show that by applying the usual finite element method one can obtain a good approximation of our minimization problem. In fact, for every $h>0$ we consider 
the usual finite element space $\mathcal{V}_{h} \subset H^{1}(\Omega)$ of piecewise linear continuous functions on a regular triangulation of $\Omega$ of size $h$, and denote

$$
\mathcal{T}_{h}:=\left\{T_{j}^{h}: 1 \leq j \leq J_{h}, \bigcup_{j} T_{j}^{h}=\Omega\right\} .
$$

Observe that in general is not possible to get $\bigcup_{j} T_{j}^{h}=\Omega$. However since $\partial \Omega$ is smooth, we can assume that $\Omega$ can be written in this way for every $h$ small enough. The error introduced is negligible. See [4] for this fact and for a general reference to the Finite Element Method.

Consider the class $\mathcal{O}^{h}$ of subsets $A_{h}$ of $\Omega$ constituted by unions of triangles $T_{j}^{h}$ of $\mathcal{T}_{h}$ that verify

- $\left|A_{h}\right| \geq \alpha$.

- There exists a triangle $T_{j}^{h} \subset A_{h}$ such that $\left|A_{h}-T_{j}^{h}\right|<\alpha$.

Observe that the numerical holes $A_{h}$ verify $\alpha \leq\left|A_{h}\right| \leq \alpha+C h^{N}$.

Our discrete optimization problem reads as follows. For each $A_{h} \in \mathcal{O}^{h}$, let

$$
\lambda_{1, h}\left(A_{h}\right):=\inf \left\{\int_{\Omega}|\nabla v|^{2}+v^{2} d x: v \in \mathcal{V}_{h},\|v\|_{L^{2}(\partial \Omega)}=1 \text { and }\left.v\right|_{A_{h}} \equiv 0\right\} .
$$

It is immediate to see (since $\mathcal{V}_{h}$ is finite dimensional) that there exists $u_{h} \in \mathcal{V}_{h}$ (a discrete eigenfunction) such that

$$
\lambda_{1, h}\left(A_{h}\right)=\int_{\Omega}\left|\nabla u_{h}\right|^{2}+u_{h}^{2} d x .
$$

As there are finitely many configurations $A_{h}$ it follows that there exists an optimal configuration $A_{h}^{*}$ such that

$$
\lambda_{1, h}\left(A_{h}^{*}\right)=\min _{\mathcal{O}^{h}} \lambda_{1, h}\left(A_{h}\right) .
$$

Our convergence result says that the numerical optimal constant and eigenfunctions approximate the continuous ones as $h \rightarrow 0$ and as a consequence, that the discrete optimal hole approximate the continuous one. We prove the following,

Theorem 3. It holds

$$
\lim _{h \rightarrow 0} \lambda_{1, h}\left(A_{h}^{*}\right)=\lambda_{1}\left(A^{*}\right) .
$$

Moreover, for any sequence $h_{j} \rightarrow 0$, there exists a subsequence $h_{j_{k}} \rightarrow 0$ and a function $u \in H^{1}(\Omega)$ such that

$$
u_{h_{j_{k}}} \rightarrow u \quad \text { strongly in } H^{1}(\Omega) \text {. }
$$

This function $u$ verifies that $A^{*}:=\{u=0\}$ is optimal for $\lambda_{1}(A)$ and $u$ is an eigenfunction for $\lambda_{1}\left(A^{*}\right)$. Finally, we have convergence of the holes,

$$
\left|A_{h_{j_{k}}}^{*} \triangle A^{*}\right| \rightarrow 0, \quad \text { as } k \rightarrow \infty,
$$

that is

$$
\mathcal{X}_{A_{h_{j}}^{*}} \rightarrow \mathcal{X}_{A^{*}} \quad \text { in } L^{1}(\Omega)
$$

In actual computations of $A^{*}$, the method described above is not plausible, since the number of elements in $\mathcal{O}^{h}$ grows exponentially as $h \rightarrow 0$.

Next we propose an algorithm to compute $\min _{\mathcal{O}^{h}} \lambda_{1, h}\left(A_{h}\right)$ and $A_{h}^{*}$ for fixed $h$. The algorithm works as follows: first, we select a set $A_{h} \in \mathcal{O}^{h}$ and compute 
$\lambda_{1, h}\left(A_{h}\right)$ (and its corresponding eigenfunction $u_{h}$ ). This is a finite dimensional eigenvalue problem. Once the discrete eigenfunction is known we compute the normal derivative at $\partial A_{h}$ and we remove some triangles from $A_{h}$ adding others (essentially preserving the measure of the hole). Following (1.3) we remove triangles with large normal derivative and add triangles adjacent to ones with small normal derivative. In this way we obtain a new set $\tilde{A}_{h}$ to iterate the procedure. More details are explained in Section 3.2.

With this procedure we find numerically the optimal hole for various domains. In the case of a ball, we observe that the optimal hole looses symmetry, as proved in Theorem 2.

1.2. Organization of the paper. The rest of the paper is organized as follows: in Section 2 we prove our shape derivative formula, (1.3) and apply it to deal with the symmetric case to prove Theorem 2. In Section 3 we prove Theorem 3 and then we propose and apply to different situations a numerical scheme to approximate the discrete optimal hole. We end the section with some comments on difficulties encountered in applying our method and possibilities for improvements.

\section{The Shape Derivative}

2.1. Computation of $\lambda_{1}^{\prime}$. In this subsection we prove the formula for $\lambda_{1}^{\prime}(A)$ given in Theorem 1.

We begin by showing that $\lambda_{1}\left(A_{t}\right)$ is differentiable at $t=0$. We remark that this fact can be obtained as a consequence of the general theory for families of operators of type A developed in [15] Chapter VII, but we provide here a direct proof of this fact by using a variant of the Implicit Function Theorem whose proof can be found, for instance, in [20].

Lemma 2.1. Let $E, F, G$ be three Banach spaces, $U \subset E$ a neighborhood of 0 and $V \subset F$ a neighborhood of $y_{0}$. Consider the applications $f$ and $g$ such that

$$
\left\{\begin{array}{l}
f: U \times V \rightarrow G \quad g: U \rightarrow V, \\
g(0)=y_{0}, \quad f(x, g(x))=0 \forall x \in U,
\end{array}\right.
$$

$f$ is differentiable at $\left(0, y_{0}\right)$,

$$
\frac{\partial f}{\partial y}\left(0, y_{0}\right): F \rightarrow G \text { is an isomorphism, }
$$

$g$ is continuous at 0.

Then, $g$ is differentiable at 0 and the derivative $\frac{\partial g}{\partial x}(0)$ verifies

$$
\frac{\partial f}{\partial x}\left(0, y_{0}\right) x+\frac{\partial f}{\partial y}\left(0, y_{0}\right) \frac{\partial g}{\partial x}(0) x=0,
$$

for every $x \in E$.

Now we use Lema 2.1 to prove that $\lambda_{1}\left(A_{t}\right)$ and its associated normalized eigenfunction $u_{t}$, are differentiable at $t=0$. 
Lemma 2.7. With the same notation and assumptions of Theorem 1 , we have that $\lambda_{1}\left(A_{t}\right)$ and its associated normalized eigenfunction $u_{t}: \mathbb{R} \rightarrow H^{1}(\Omega)$ are differentiable at $t=0$.

Proof. We have

$$
\int_{\Omega} \nabla u_{t} \nabla \varphi+u_{t} \varphi d x=\lambda_{1}\left(A_{t}\right) \int_{\partial \Omega} u_{t} \varphi d S .
$$

Making the change of variables $x=y+t V(y)$ and calling $\bar{u}_{t}(y)=u_{t}(y+t V(y))$, $\bar{\varphi}(y)=\varphi(y+t V(y))$, after integration by parts we obtain

$$
\int_{\Omega} a_{t}(x) \nabla \bar{u}_{t} \nabla \bar{\varphi}+b_{t}(x) \bar{u}_{t} \bar{\varphi} d x-\lambda_{1}\left(A_{t}\right) \int_{\partial \Omega} \bar{u}_{t} \bar{\varphi} d S=0,
$$

where $a_{t}(x):=\operatorname{det}(I d+t D V) \cdot(I d+t D V)^{-1} \cdot\left[(I d+t D V)^{-1}\right]^{t}$ and $b_{t}(x):=\operatorname{det}(I d+$ $t D V)$. Observe that $\left.a_{t}(x)\right|_{\partial \Omega}=I, a_{0}(x)=I, b_{0}(x)=1$ and that the application $\varphi \mapsto \bar{\varphi}$ is an isomorphism between $H_{A_{t}}^{1}(\Omega)$ and $H_{A}^{1}(\Omega)$.

Recall that, since $V$ has compact support,

$$
\int_{\partial \Omega} \bar{u}_{t}^{2} d S=1
$$

Now let

$$
g(t)=\left(\lambda_{1}\left(A_{t}\right), \bar{u}_{t}\right) \in \mathbb{R} \times H_{A}^{1}(\Omega)
$$

and $f: \mathbb{R} \times \mathbb{R} \times H_{A}^{1}(\Omega) \rightarrow\left(H_{A}^{1}(\Omega)\right)^{\prime} \times \mathbb{R}$, where $\left(H_{A}^{1}(\Omega)\right)^{\prime}$ is the (topological) dual space of $H_{A}^{1}(\Omega)$, given by $f=\left(f_{1}, f_{2}\right)$,

$$
\begin{aligned}
f_{1}(t, \lambda, v) \phi & :=\int_{\Omega} a_{t}(x) \nabla v \nabla \phi+b_{t}(x) v \phi d x-\lambda \int_{\partial \Omega} v \phi d S, \\
f_{2}(t, \lambda, v) & :=\int_{\partial \Omega} v^{2} d S-1 .
\end{aligned}
$$

The continuity of $g$ is proved in [11], Theorem 1.6 and one can easily check hypotheses (2.2) and (2.3) of Lemma 2.1. It remains to check (2.4).

First, we compute

$$
\begin{aligned}
\frac{\partial f_{1}}{\partial(\lambda, v)}\left(0, \lambda_{1}(A), u\right)(\mu, w) \phi & =\int_{\Omega} \nabla w \nabla \phi+w \phi d x-\int_{\partial \Omega}\left(\lambda_{1}(A) w+\mu u\right) \phi d S \\
\frac{\partial f_{2}}{\partial(\lambda, v)}\left(0, \lambda_{1}(A), u\right)(\mu, w) & =2 \int_{\partial \Omega} u w d S .
\end{aligned}
$$

Now, the fact that $\partial f /\left.\partial(\lambda, v)\right|_{\left(0, \lambda_{1}(A), u\right)}$ is an isomorphism follows by the Fredholm alternative applied to the operator $S:\left(H_{A}^{1}(\Omega)\right)^{\prime} \rightarrow\left(H_{A}^{1}(\Omega)\right)^{\prime}$ given by $S h:=v$ where $v$ is the solution to

$$
\int_{\Omega} \nabla v \nabla \phi+v \phi d x-\lambda_{1}(A) \int_{\partial \Omega} v \phi=\langle h, \phi\rangle,
$$

for every $\phi \in H_{A}^{1}(\Omega)$. Here, $\langle\cdot, \cdot\rangle$ denotes the duality pairing between $\left(H_{A}^{1}(\Omega)\right)^{\prime}$ and $H_{A}^{1}(\Omega)$.

In fact, first one uniquely determines $\mu$ such that $h \perp u$ and so $w=w_{0}+s u$ for any $s \in \mathbb{R}$ and finally one uniquely determines $s$ so that $2 \int_{\partial \Omega} u w d S=r$ for a 
given $r \in \mathbb{R}$. Observe that the inclusion of $H_{A}^{1}(\Omega) \cap\left(H_{0}^{1}(\Omega)\right)^{\perp}$ in $\left(H_{A}^{1}(\Omega)\right)^{\prime}$ is given by $v \mapsto \varphi_{v}$ where

$$
\left\langle\varphi_{v}, \phi\right\rangle:=\int_{\partial \Omega} v \phi d S
$$

This proves that $g$ is differentiable at $t=0$ and therefore $\lambda_{1}\left(A_{t}\right)$ and $u_{t}$ are differentiable at $t=0$.

Proof of Theorem 1. To find the expression for $\lambda_{1}^{\prime}(A)$ in Theorem 1, we first look for a problem that is satisfied by the derivative $u^{\prime}=\left.\frac{\partial}{\partial t} u_{t}\right|_{t=0}$, namely

$$
\begin{cases}\Delta u^{\prime}=u^{\prime} & \text { in } \Omega \backslash \bar{A} \\ \frac{\partial u^{\prime}}{\partial \nu}=\lambda_{1}^{\prime}(A) u+\lambda_{1}(A) u^{\prime} & \text { on } \partial \Omega \\ u^{\prime}=-\frac{\partial u}{\partial \nu}\langle V, \nu\rangle, & \text { on } \partial A .\end{cases}
$$

Next, we use this expression together with the weak form of the equation verified by $u,(1.1)$, to compute the formula for the derivative $\lambda_{1}^{\prime}(A),(1.3)$.

In Chapter 5 of [14], a similar problem is treated: Let $U_{t}:=(I d+t V)(U)$, where $U \subset \mathbb{R}^{N}$ is a bounded domain, and $v_{t} \in H_{0}^{1}\left(U_{t}\right)$ be the weak solution of

$$
\begin{cases}-\Delta v+v=f & \text { in } U_{t} \\ v=0 & \text { on } \partial U_{t}\end{cases}
$$

where $f \in L^{2}\left(\mathbb{R}^{N}\right)$. Then $v_{t}$ is differentiable at $t=0$ and if $v:=v_{0} \in H^{2}(\Omega)$ then $v^{\prime}$ is the solution of

$$
\begin{cases}-\Delta v^{\prime}+v^{\prime}=0 & \text { in } U \\ v^{\prime}=-\frac{\partial v}{\partial \nu}\langle V, \nu\rangle & \text { on } \partial U\end{cases}
$$

Using the fact that $\lambda_{1}\left(A_{t}\right)$ is differentiable at $t=0$ and arguing exactly as in [14] it can be proved in our case that $u_{t}$ is differentiable at $t=0$ and that $u^{\prime}$ verifies (2.8).

To find the expression for $\lambda_{1}^{\prime}(A)$ we observe that from $\Delta u^{\prime}=u^{\prime}$ and $\Delta u=u$ we obtain

$$
\int_{\partial \Omega} \frac{\partial u^{\prime}}{\partial \nu} u d S=\int_{\partial \Omega} \frac{\partial u}{\partial \nu} u^{\prime} d S+\int_{\partial A} \frac{\partial u}{\partial \nu} u^{\prime} d S
$$

Hence,

$$
\lambda_{1}^{\prime}(A) \int_{\partial \Omega} u^{2} d S+\lambda_{1}(A) \int_{\partial \Omega} u u^{\prime} d S=\lambda_{1}(A) \int_{\partial \Omega} u u^{\prime} d S+\int_{\partial A} \frac{\partial u}{\partial \nu} u^{\prime} d S .
$$

Using that we have normalized $u$ by (1.2), that is $\int_{\partial \Omega} u^{2} d S=1$, we get

$$
\lambda_{1}^{\prime}(A)=-\int_{\partial A}\left(\frac{\partial u}{\partial \nu}\right)^{2}\langle V, \nu\rangle d S
$$

as we wanted to prove. 
2.2. Applications to a symmetric domain: The ball. In this subsection we take a symmetric domain $\Omega=B_{R}$, the ball of radius $R$ centered at 0 , and prove that the optimal hole is not completely symmetric.

Proof of Theorem 2. First we prove (1)-(a), which is a consequence of Theorem 1. Then we consider $A=B_{r}$. Since the first eigenvalue $\lambda_{1}(A)$ is simple (see [18]), it follows that $u$ is radial. Hence there exists a constant $C$ such that at the boundary of the hole we have,

$$
\frac{\partial u}{\partial \nu}=C
$$

Therefore, using that we are dealing with deformations $V$ that preserves the volume of the hole, we get

$$
\lambda_{1}^{\prime}(A)=-\int_{\partial A}\left(\frac{\partial u}{\partial \nu}\right)^{2}\langle V, \nu\rangle d S=-C^{2} \int_{\partial A}\langle V, \nu\rangle d S=C^{2} \int_{A} \operatorname{div}(V) d x=0 .
$$

To prove (1)-(b) we need a Lemma that concerns $\lambda_{1}(R, r):=\lambda_{1}\left(B_{r}\right)$. We cannot find this optimal constant explicitly, nevertheless it verifies a differential equation.

Lemma 2.9. Let $R>r$, then $\lambda_{1}(R, r):=\lambda_{1}\left(B_{r}\right)$ verifies the differential equation

$$
\frac{\partial \lambda_{1}}{\partial R}=-\frac{N-1}{R} \lambda_{1}+1-\lambda_{1}^{2}
$$

with the condition

$$
\left.\lambda_{1}\right|_{R=r}=+\infty .
$$

Proof. Let $\varphi(s)$ be a solution of

$$
\left\{\begin{array}{l}
\varphi^{\prime \prime}+\frac{N-1}{s} \varphi^{\prime}=\varphi \quad \text { in } s>r \\
\varphi^{\prime}(r)=1 \\
\varphi(r)=0 .
\end{array}\right.
$$

Hence $u(x):=\varphi(|x|)$ is a radial solution of $\Delta u=u$ in $B_{R} \backslash \overline{B_{r}}$ with $\left.u\right|_{\partial B_{r}}=0$.

Therefore, by the simplicity of $\lambda_{1}, u$ is the eigenfunction associated with $\lambda_{1}$ for every $R>r$. Thus

$$
\varphi^{\prime}(R)=\lambda_{1} \varphi(R) .
$$

From this equation we obtain an expression for the derivative of $\lambda_{1}$. Namely,

$$
\frac{\partial \lambda_{1}}{\partial R}=\frac{\partial\left(\varphi^{\prime} / \varphi\right)(R)}{\partial R}=\frac{\varphi^{\prime \prime}}{\varphi}(R)-\left(\frac{\varphi^{\prime}}{\varphi}\right)^{2}(R) .
$$

Now we observe that, by (2.12),

$$
\frac{\varphi^{\prime \prime}}{\varphi}(R)=\frac{-\frac{N-1}{R} \varphi^{\prime}+\varphi}{\varphi}(R)=-\frac{N-1}{R} \lambda_{1}+1,
$$

Hence we get,

$$
\frac{\partial \lambda_{1}}{\partial R}=-\frac{N-1}{R} \lambda_{1}+1-\lambda_{1}^{2} .
$$

To check the condition (2.11) we proceed as in [11]. We claim that

$$
\lambda_{1}\left(B_{r}\right) \rightarrow \infty \quad \text { as } \quad \varepsilon=R-r \rightarrow 0 .
$$


In fact, let $u_{\varepsilon} \in H^{1}\left(B_{R}\right)$ be an extremal for $\lambda_{1}\left(B_{r}\right)$ normalized so that

$$
\left\|u_{\varepsilon}\right\|_{L^{2}\left(\partial B_{R}\right)}=1 \text {. }
$$

Thus, $u_{\varepsilon}=0$ in $B_{r}$ and so $u_{\varepsilon} \rightarrow 0$ a.e. $B_{R}$ as $\varepsilon \rightarrow 0$.

If $\lambda_{1}\left(B_{r}\right)$ were bounded then, up to a subsequence, there exists a function $u \in$ $H^{1}\left(B_{R}\right)$ such that

$$
\begin{array}{ll}
u_{\varepsilon} \rightarrow u & \text { weakly in } H^{1}\left(B_{R}\right), \\
u_{\varepsilon} \rightarrow u & \text { strongly in } L^{2}\left(B_{R}\right) \text { and a.e. in } B_{R}, \\
u_{\varepsilon} \rightarrow u & \text { strongly in } L^{2}\left(\partial B_{R}\right) .
\end{array}
$$

Since $u_{\varepsilon} \rightarrow 0$ a.e. $B_{R}$ and $\left\|u_{\varepsilon}\right\|_{L^{2}(\partial \Omega)}=1$ we arrive to a contradiction.

From this lemma we get the following corollary.

Corollary 2.13. The function $\lambda_{1}=\lambda_{1}(R, r)$ verifies

$$
\lambda_{1}^{2}+\lambda_{1} \frac{N-1}{r}>1, \quad \text { for all } R>r .
$$

Proof. We begin differentiating (2.10) to obtain

$$
\frac{\partial^{2} \lambda_{1}}{\partial^{2} R}=-\frac{N-1}{R} \frac{\partial \lambda_{1}}{\partial R}+\frac{N-1}{R^{2}} \lambda_{1}-2 \lambda_{1} \frac{\partial \lambda_{1}}{\partial R} .
$$

Observe that, since $\lambda_{1}>0$, at any critical point $\left(\lambda_{1}^{\prime}=0\right)$ we have $\lambda_{1}^{\prime \prime}>0$. Hence $\lambda_{1}$ has at most one critical point, which is a minimum. If $\lambda_{1}$ has a minimum, when is reached we get from (2.10) that

$$
1=\frac{N-1}{R} \lambda_{1}+\lambda_{1}^{2}<\frac{N-1}{r} \lambda_{1}+\lambda_{1}^{2}
$$

If $\lambda_{1}$ has no critical points then, since $\lambda_{1} \rightarrow+\infty$ as $R \rightarrow r$ we get that the conclusion holds at least for $R$ close to $r$. Now we observe that in this case $\lambda_{1}$ is strictly decreasing as a function of $R$ and, from the equation (2.10), it verifies $\lim _{R \rightarrow \infty} \lambda_{1}=1$.

Now we can proceed with the end of the proof of Theorem 2. Let $\phi$ be the solution of

$$
\left\{\begin{array}{l}
\phi^{\prime \prime}+\frac{N-1}{s} \phi^{\prime}=\phi \quad \text { in } r<s<R, \\
\phi(R)=1, \\
\phi(r)=0 .
\end{array}\right.
$$

The function $v(x):=\phi(|x|)$ is an extremal for our minimization problem.

Now let us move this symmetric configuration in the $x_{1}$ direction. To this end, first extend $\phi$ to the whole $\mathbb{R}$ extending by zero to $(0, r)$ and as a solution of the equation $\phi^{\prime \prime}+\frac{N-1}{s} \phi^{\prime}=\phi$ with $\phi(R)=1$, to $(R,+\infty)$. Then $v$ is extended to the whole $\mathbb{R}^{N}$. Let

and define

$$
x_{t}=\left(x_{1}-t, x_{2}, \ldots, x_{N}\right),
$$

$$
U(t)(x)=v\left(x_{t}\right) .
$$

This function $U$ vanishes in $A_{t}:=B_{r}\left(t e_{1}\right)$ a subset of $\Omega=B_{R}$ of the same area of $B_{r}$ for every $t$ small. 
Now, let us compute the derivatives of

$$
\Theta(t):=\frac{\int_{B_{R}}|\nabla U(t)|^{2}+|U(t)|^{2} d x}{\int_{\partial B_{R}}|U(t)|^{2} d S} .
$$

We have,

$$
\Theta(0)=\lambda_{1}\left(B_{r}\right)
$$

Observe that, since $\Theta$ is an even function, we have

$$
\Theta^{\prime}(0)=0 \text {. }
$$

To obtain $\Theta^{\prime \prime}(0)$ we denote

$$
f(t):=\int_{B_{R}}|\nabla U(t)|^{2}+|U(t)|^{2} d x \quad \text { and } \quad g(t):=\int_{\partial B_{R}}|U(t)|^{2} d S .
$$

Hence $\Theta^{\prime \prime}(0)=\left.g^{-3}\left(f^{\prime \prime} g^{2}-f g g^{\prime \prime}-2 f^{\prime} g^{\prime} g+2 f\left(g^{\prime}\right)^{2}\right)\right|_{t=0}$. Next we compute these terms:

$$
\begin{aligned}
f(0) & =\lambda_{1}\left|\partial B_{R}\right|, & g(0) & =\left|\partial B_{R}\right| \\
f^{\prime}(0) & =-\int_{B_{R}} \frac{\partial}{\partial x_{1}}\left(|\nabla v|^{2}+v^{2}\right) d x, & g^{\prime}(0) & =-2 \int_{\partial B_{R}} \frac{\partial v}{\partial x_{1}} d S \\
f^{\prime \prime}(0) & =\int_{B_{R}} \frac{\partial^{2}}{\partial x_{1}^{2}}\left(|\nabla v|^{2}+v^{2}\right) d x, & g^{\prime \prime}(0) & =2 \int_{\partial B_{R}}\left(\frac{\partial v}{\partial x_{1}}\right)^{2}+\frac{\partial^{2} v}{\partial x_{1}^{2}} d S .
\end{aligned}
$$

By means of Green's Theorem we obtain

$$
\begin{aligned}
f^{\prime}(0) & =-\int_{\partial B_{R}}\left(|\nabla v|^{2}+v^{2}\right) \nu_{1} d S \\
f^{\prime \prime}(0) & =\int_{\partial B_{R}} \frac{\partial}{\partial x_{1}}\left(|\nabla v|^{2}+v^{2}\right) \nu_{1} d S .
\end{aligned}
$$

Since $v$ is radial, we get

$$
f^{\prime}(0)=g^{\prime}(0)=0
$$

Summing up,

$$
\Theta^{\prime \prime}(0)=\left|\partial B_{R}\right|^{-1}\left(\int_{\partial B_{R}} \frac{\partial}{\partial x_{1}}\left(|\nabla v|^{2}+v^{2}\right) \nu_{1} d S-2 \lambda_{1} \int_{\partial B_{R}}\left(\frac{\partial v}{\partial x_{1}}\right)^{2}+\frac{\partial^{2} v}{\partial x_{1}^{2}} d S\right) .
$$

Observe that since $v$ is radial we can replace $x_{1}$ by any $x_{i}, i=1, \ldots, N$. Therefore

$$
\Theta^{\prime \prime}(0)=\frac{1}{N\left|\partial B_{R}\right|}\left(\int_{\partial B_{R}} \frac{\partial}{\partial \nu}\left(|\nabla v|^{2}+v^{2}\right) d S-2 \lambda_{1} \int_{\partial B_{R}}|\nabla v|^{2}+\Delta v d S\right) .
$$

Now, we compute

$$
\left.\frac{\partial}{\partial \nu}\left(|\nabla v|^{2}+v^{2}\right)\right|_{\partial B_{R}}=2 \phi^{\prime}(R)\left(\phi^{\prime \prime}(R)+\phi(R)\right)=2 \lambda_{1}\left(2-\frac{N-1}{R} \lambda_{1}\right)
$$

and

$$
\left.\left(|\nabla v|^{2}+\Delta v\right)\right|_{\partial B_{R}}=\left(\phi^{\prime}(R)\right)^{2}+\phi(R)=\lambda_{1}^{2}+1 .
$$


Finally,

$$
\Theta^{\prime \prime}(0)=\frac{2}{N} \lambda_{1}\left[1-\frac{N-1}{R} \lambda_{1}-\lambda_{1}^{2}\right] .
$$

By (2.14), we get that

$$
\Theta^{\prime \prime}(0)<0 .
$$

Hence 0 is a strict local maxima of $\Theta$. Now,

$$
\lambda_{1}\left(B_{r}\right)=\Theta(0)>\Theta(t) \geq \lambda_{1}\left(B_{r}\left(t e_{1}\right)\right),
$$

for every $t$ small. Therefore the symmetric centered hole with a radial extremal is not an optimal configuration.

To end the proof we show that the half ball is not an optimal configuration either. To prove this fact, we argue by contradiction. Let us assume that $A^{*}=$ $B(0,1) \cap\left\{x_{1}<0\right\}$ is an optimal hole. Then the associated eigenfunction verifies $u=0, u_{x_{1}}=$ constant on $\left\{x_{1}=0\right\}$, and $\Delta u=u$ in $B(0,1) \cap\left\{x_{1}>0\right\}$. Let us consider the function

$$
v(x)=a\left(e^{x_{1}}-e^{-x_{1}}\right) .
$$

This function verifies $\Delta v=v_{x_{1} x_{1}}=v$ in $B(0,1) \cap\left\{x_{1}>0\right\}$ and on $x_{1}=0$, $v=0$ and $v_{x_{1}}=$ constant (that we may assume that is the same as the constant for $u_{x_{1}}$ choosing $a$ appropriately). Therefore $w=(u-v)$ satisfies $\Delta w=w$ in $B(0,1) \cap\left\{x_{1}>0\right\}, w=0$ in $B(0,1) \cap\left\{x_{1}<0\right\}$ and $w_{x_{1}}=0$ on $x_{1}=0$. Hence $w$ vanishes in the whole ball and then $u(x)=v(x)$. But now we observe that $v(x)$ does not verify $\partial v / \partial \nu=\lambda v$ on the boundary $\left\{|x|=1, x_{1}>0\right\}$. This contradiction proves that taking half the ball as the hole is not optimal.

Remark 2.15. The proof above shows that the centered ball is in fact a local maxima among balls of the same measure.

Remark 2.16. It can be checked, arguing as in the proof of the preceding Theorem, that for every $\gamma, B \cap\left\{x_{1}>\gamma\right\}$ is not optimal among sets of the same measure.

\section{Numerical approximation of the optimal hole}

3.1. Finite Element approximation. In this subsection we prove that the FEM described in the introduction gives a good approximation procedure for the minimization problem.

Proof of Theorem 3. Let $A_{h}^{*}$ be an optimal configuration and $u_{h}$ an associated eigenfunction. Then

$$
\left\|u_{h}\right\|_{H^{1}(\Omega)}^{2}=\lambda_{1, h}\left(A_{h}^{*}\right) \leq \lambda_{1, h}\left(\left(A_{0}\right)_{h}\right)<C,
$$

where $\left(A_{0}\right)_{h}$ is a discretization of a fixed regular set $A_{0} \subset \Omega$ of measure $\alpha$. Therefore, we have that $\left\{u_{h}\right\}_{h>0}$ is bounded in $H^{1}(\Omega)$. So, for any sequence $h_{j} \rightarrow 0$, there exists a subsequence $h_{j_{k}} \rightarrow 0$ and a function $u \in H^{1}(\Omega)$ such that

$$
\begin{array}{ll}
u_{h_{j_{k}}} \rightarrow u & \text { weakly in } H^{1}(\Omega), \\
u_{h_{j_{k}}} \rightarrow u & \text { strongly in } L^{2}(\Omega), \\
u_{h_{j_{k}}} \rightarrow u & \text { strongly in } L^{2}(\partial \Omega), \\
u_{h_{j_{k}}} \rightarrow u & \text { a.e. in } \Omega .
\end{array}
$$


By the above mentioned convergence, we have that $\|u\|_{L^{2}(\partial \Omega)}=1$ and

$$
|\{u=0\}| \geq \limsup _{k \rightarrow \infty}\left|\left\{u_{h_{j_{k}}}=0\right\}\right| \geq \lim _{k \rightarrow \infty}\left|A_{h_{j_{k}}}^{*}\right|=\alpha .
$$

Now,

$$
\liminf _{k \rightarrow \infty} \lambda_{1, h_{j_{k}}}\left(A_{h_{j_{k}}}^{*}\right) \geq \int_{\Omega}|\nabla u|^{2}+u^{2} d x \geq\left\|u_{\alpha}\right\|_{H^{1}(\Omega)}^{2} .
$$

Here $u_{\alpha} \in H^{1}(\Omega)$ is an extremal, i.e. $\left|\left\{u_{\alpha}=0\right\}\right| \geq \alpha,\left\|u_{\alpha}\right\|_{L^{2}(\partial \Omega)}=1$ and $\left\|u_{\alpha}\right\|_{H^{1}(\Omega)}^{2} \leq\|w\|_{H^{1}(\Omega)}^{2}$ for every $w$ that vanishes in a hole of measure $\alpha$. It is proved in [11] that $\left|\left\{u_{\alpha}=0\right\}\right|=\alpha$ and in [12] that $u_{\alpha}$ is locally Lipschitz continuous.

Given $\varepsilon>0$, for any $h$ small enough $\left(h<h_{0}(\varepsilon)\right)$, let $v_{h} \in \mathcal{V}_{h}$ be such that $\left\|u_{\alpha}-v_{h}\right\|_{H^{1}(\Omega)}<\varepsilon,\left\|v_{h}\right\|_{L^{2}(\partial \Omega)}=1$ and such that there exists a set $A_{h} \in \mathcal{O}^{h}$ with $A_{h} \subset\left\{v_{h}=0\right\}$.

This function $v_{h}$ can be constructed as follows: First, we define $\bar{v}_{h}$ in this way, for every triangle $T_{j}^{h}$ with $T_{j}^{h} \cap\left\{u_{\alpha}=0\right\} \neq \emptyset$ we put $\left.\bar{v}_{h}\right|_{T_{j}^{h}} \equiv 0$ and in the rest of the nodes of the mesh we put $\bar{v}_{h}=u_{\alpha}$. This defines a unique function $\bar{v}_{h} \in \mathcal{V}_{h}$ that verifies

$$
\left\|u_{\alpha}-\bar{v}_{h}\right\|_{H^{1}(\Omega)}<\delta
$$

for every $h<h_{0}(\delta)$. This is a standard interpolation error estimate since $u_{\alpha}$ is regular in $\Omega \backslash \overline{A_{\alpha}}$. See [4].

Observe that, as $\left\|u_{\alpha}\right\|_{L^{2}(\partial \Omega)}=1$, we have

$$
1-C \delta<\left\|\bar{v}_{h}\right\|_{L^{2}(\partial \Omega)}<1+C \delta,
$$

hence, $v_{h}:=\bar{v}_{h} /\left\|\bar{v}_{h}\right\|_{L^{2}(\partial \Omega)}$ satisfies all the above requirements.

Now, we have

$$
\int_{\Omega}\left|\nabla v_{h}\right|^{2}+v_{h}^{2} d x<\varepsilon+\int_{\Omega}\left|\nabla u_{\alpha}\right|^{2}+u_{\alpha}^{2} d x=\varepsilon+\left\|u_{\alpha}\right\|_{H^{1}(\Omega)}^{2},
$$

from where $\lambda_{1, h}\left(A_{h}^{*}\right) \rightarrow\left\|u_{\alpha}\right\|_{H^{1}(\Omega)}^{2}$ as $h \rightarrow 0$. Now, as $u_{h_{j_{k}}} \rightarrow u$ weakly in $H^{1}(\Omega)$ and

$$
\left\|u_{h_{j_{k}}}\right\|_{H^{1}(\Omega)}^{2}=\lambda_{1, h_{j_{k}}}\left(A_{h_{j_{k}}}^{*}\right) \rightarrow\|u\|_{H^{1}(\Omega)}^{2},
$$

it follows that $u_{h_{j_{k}}} \rightarrow u$ strongly in $H^{1}(\Omega)$.

Finally, as $u$ is an extremal, by [11] we have $|\{u=0\}|=\alpha$.

The fact that $\left|A^{*} \triangle A_{h}^{*}\right| \rightarrow 0$ as $h \rightarrow 0$ is a consequence of Lemma 3.1 below. This finishes the proof.

Lemma 3.1. Let $f_{n}, f: \Omega \rightarrow \mathbb{R}_{\geq 0}$ be measurable functions such that $f_{n}(x) \rightarrow f(x)$ a.e. in $\Omega$. Suppose that $\left|\left\{f_{n}=0\right\}\right| \rightarrow|\{f=0\}|$. Then

$$
\left|\left\{f_{n}=0\right\} \triangle\{f=0\}\right| \rightarrow 0, \quad \text { as } n \rightarrow \infty .
$$

Proof. By Egoroff's Theorem, given $\varepsilon>0$, there exists a closed set $C_{\varepsilon} \subset \Omega$ such that

$$
\left|C_{\varepsilon}\right|<\varepsilon \quad \text { and } \quad f_{n} \rightarrow f \quad \text { uniformly in } \Omega \backslash C_{\varepsilon} .
$$

We call $E_{\varepsilon}:=\Omega \backslash C_{\varepsilon}$. By the uniform convergence, for any $\delta>0$,

$$
\left\{f_{n}=0\right\} \cap E_{\varepsilon} \subset\{f \leq \delta\} \cap E_{\varepsilon},
$$

if $n$ is large enough. 
Now,

$$
\{f=0\} \backslash\left\{f_{n}=0\right\} \subset\left(\left(\{f \leq \delta\} \backslash\left\{f_{n}=0\right\}\right) \cap E_{\varepsilon}\right) \cup C_{\varepsilon} .
$$

Therefore, by (3.2), we obtain

$$
\left|\{f=0\} \backslash\left\{f_{n}=0\right\}\right| \leq|\{f \leq \delta\}|-\left|\left\{f_{n}=0\right\}\right|+2 \varepsilon .
$$

Taking limit first as $n \rightarrow \infty$ and then as $\delta \rightarrow 0$ we obtain

$$
\limsup _{n \rightarrow \infty}\left|\{f=0\} \backslash\left\{f_{n}=0\right\}\right| \leq 2 \varepsilon
$$

SO

$$
\lim _{n \rightarrow \infty}\left|\{f=0\} \backslash\left\{f_{n}=0\right\}\right|=0
$$

Finally, by (3.2),

$$
\left\{f_{n}=0\right\} \backslash\{f=0\} \subset\left((\{f \leq \delta\} \backslash\{f=0\}) \cap E_{\varepsilon}\right) \cup C_{\varepsilon} .
$$

As before, we obtain,

$$
\left|\left\{f_{n}=0\right\} \backslash\{f=0\}\right| \leq|\{f \leq \delta\}|-|\{f=0\}|+2 \varepsilon,
$$

from where it follows that

$$
\lim _{n \rightarrow \infty}\left|\left\{f_{n}=0\right\} \backslash\{f=0\}\right|=0
$$

The proof is now complete.

3.2. The numerical algorithm. In this subsection we describe a numerical scheme, based on our formula for the shape derivative with respect to the hole, to approximate the optimal hole and the optimal extremal. Recall that we have to minimize $\lambda_{1, h}\left(A_{h}\right), A_{h} \in \mathcal{O}^{h}$.

The Algorithm.

(1) Choose an initial hole $A_{h}^{0}$. This hole must verify

- $\left|A_{h}\right| \geq \alpha$.

- There exists a triangle $T_{j}^{h} \subset A_{h}$ such that $\left|A_{h}-T_{j}^{h}\right|<\alpha$.

(2) Compute $\lambda_{1, h}\left(A_{h}^{0}\right)$ and the extremal $u_{h}^{0}$. Observe that this is a finite dimensional eigenvalue problem.

(3) Compute the normal derivative at the boundary of $A_{h}^{0}$.

(4) Sort the faces of the triangles. They are sorted according to the sizes of the computed normal derivatives. We call the sorted faces $e_{1}, \ldots, e_{K}$ and the triangles containing them $T_{1}, \ldots, T_{K}, \tilde{T}_{1}, \ldots, \tilde{T}_{K}$. The first ones belonging to $A_{h}^{0}$ and the last ones to $\Omega \backslash A_{h}^{0}$.

(5) While $\left|A_{h}^{0}\right|>\alpha, A_{h}^{0}=A_{h}^{0}-T_{K-j}, j=j+1$. We remove the triangles with larger normal derivative until the measure of the hole lies below $\alpha$.

(6) While $\left|A_{h}^{0}\right|<\alpha, A_{h}^{0}=A_{h}^{0} \cup \tilde{T}_{i}, i=i+1$. We add triangles to the hole in regions of the boundary where the normal derivative is small.

(7) Update the hole: $A_{h}^{1}:=A_{h}^{0}$.

Observe that this new hole $A_{h}^{1}$ verifies the conditions imposed to $A_{h}^{0}$. Hence, we can iterate this procedure from (2). 
FIGURE 1. L-shaped domain

Figure 2. Two balls with handle

3.3. Numerical results. In this subsection we use the algorithm described in the previous subsection to different situations in order to see what the optimal hole looks like. To compute $\lambda_{1, h}\left(A_{h}^{0}\right)$ and the extremal $u_{h}^{0}$ we use the minimization routine fmincon from MATLAB.

In the first column of Figures 1 to 3 the initial domain $A_{h}$ used to start the method is shown. The second and third columns show the final (best) hole and the corresponding extremal.

In Figure 1 the L-shaped domain is composed by three squares of area 1, we have used $\alpha=1 / 2$.

In Figure 2 the domain is composed by two balls with a handle. Each ball has radius 1 and we take $\alpha=\pi$, the area of one of the balls.

In Figure 3 the domain is the unit ball. In this case we take $\alpha=\pi / 2$, half the measure of the ball. As can be seen the optimal hole is neither a centered ball nor half the ball. It can also be appreciated that the (numerical) best hole is approximately spherically symmetric.

In Figure 4, we show the evolution of $\lambda_{1, h}\left(A_{h}\right)$ (with fixed $h$ ) as the algorithm iterates. As can be seen it is not true that $\lambda_{1, h}\left(A_{h}\right)$ decreases from one step to another, but it does in the long run.

Next we analyze the behavior of the approximations of $A_{h}^{*}, \lambda_{1, h}\left(A_{h}^{*}\right)$ by our method as $h \rightarrow 0$, which is shown in Table 1 and Figure 5. We use for this computations the L-shaped domain with $\alpha=1$. The first column of the table shows the value of $h$, the mesh parameter, the second one, the area of the numerical 
FiguRE 3. The ball

FiguRE 4. Behavior of the eigenvalues as the algorithm evolves

\begin{tabular}{|c|c|c|}
\hline$h$ & $\left|A_{h}^{*}\right|$ & $\lambda_{1, h}\left(A_{h}^{*}\right)$ \\
\hline 0.80 & 1.0000 & 0.58058 \\
\hline 0.50 & 1.0018 & 0.57848 \\
\hline 0.25 & 0.9729 & 0.54091 \\
\hline 0.10 & 1.0004 & 0.50227 \\
\hline
\end{tabular}

TABLE 1. Numerical optimal eigenvalues

optimal hole $A_{h}^{*}$ and the last one, the value of the numerical optimal constant $\lambda_{1, h}\left(A_{h}^{*}\right)$. In Figure 5 the numerical optimal holes are drawn.

3.4. Final comments. Let us mention some difficulties and possible improvements for the algorithm.

The algorithm as described above, may develop cycles (in particular when near the optimal hole) that may prevent convergence. In order to avoid this problem several ad hoc strategies can be adopted. We choose not to allow the method to 


$$
h=0.80 \quad h=0.50 \quad h=0.25 \quad h=0.1
$$

Figure 5. Behavior of the numerical optimal hole as $h \rightarrow 0$

add a triangle to the hole that has been removed from it some fixed number of steps ago.

It can be observed from the numerical simulations that the problem of finding the discrete optimal hole for fixed $h$ has many spurious minima. By a spurious minima we mean that there exists non-optimal configurations with the following property: if we remove any triangle of the hole and add another one on any part of the boundary of the hole, the new eigenvalue is larger than the previous one. When running the algorithm one should be aware of this fact.

Due to above mentioned facts, in general, is very hard to prove convergence results for these kind of problems. Nevertheless, it can be appreciated convergence of the algorithm for fixed $h$ (see Figures 1-4) and convergence of the computed optimal holes as $h \rightarrow 0$ (see Table 1 and Figure 5 ). The results in Theorem 3 give the convergence of the discrete optimal holes to the continuous one as the mesh size $h$ goes to zero.

For fixed $h$ and a fixed hole $A_{h}$, different methods can be applied to compute the discrete eigenvalue. Methods specially designed for eigenvalue problems can be used. The method we use here has the advantage of being easily implemented through the iterations and of being more flexible when dealing with nonlinear problems where eigenvalue techniques do not apply.

The method, as described above, replaces very few triangles of the hole in each step. That is not efficient if we are far from the optimal hole. So, it is better to replace several triangles in the firsts steps of the algorithm and decrease the quantity of replaced triangles as the algorithm iterates.

A stopping criteria should be established to decide whether to stop the algorithm. There are many possibilities to do this, let us mention two of them

- If $\lambda_{1, h}\left(A_{h}\right)$ decreases less than a fixed tolerance from one step to another (or in several steps).

- If the method tries to add triangles to the hole that have been removed a few steps ago (it can be observed in numerical simulations that this happens when we are close to the optimal hole).

This method seems better suited to find the location of the hole rather than the fine resolution of its boundary. Adaptivity (near the boundary of the optimal computed hole) should be used to achieve this goal. 


\section{ACKNOWLEDGEMENTS}

We want to thank the anonymous referee for the careful reading of the manuscript, the constructive criticism and several suggestions that help us to improve the paper.

\section{REFERENCES}

[1] T. Aubin. Équations différentielles non linéaires et le problème de Yamabe concernant la courbure scalaire. J. Math. Pures et Appl., 55 (1976), 269-296.

[2] R.J. Biezuner. Best constants in Sobolev trace inequalities. Nonlinear Analysis, 54 (2003), $575-589$.

[3] A. Cherkaev and E. Cherkaeva. Optimal design for uncertain loading condition. Homogenization, 193-213, Ser. Adv. Math. Appl. Sci., 50, World Sci. Publishing, River Edge, NJ, 1999.

[4] P. Ciarlet. The finite element method for elliptic problems. North Holland, (1978).

[5] O. Druet and E. Hebey. The AB program in geometric analysis: sharp Sobolev inequalities and related problems. Mem. Amer. Math. Soc. 160 (761) (2002).

[6] M. del Pino and C. Flores. Asymptotic behavior of best constants and extremals for trace embeddings in expanding domains. Comm. Partial Differential Equations, 26 (11-12) (2001), 2189-2210.

[7] J. F. Escobar. Sharp constant in a Sobolev trace inequality. Indiana Math. J., 37 (3) (1988), $687-698$.

[8] J. Fernández Bonder, E. Lami Dozo and J.D. Rossi. Symmetry properties for the extremals of the Sobolev trace embedding. Ann. Inst. H. Poincaré. Anal. Non Linéaire, 21 (2004), no. 6, 795-805.

[9] J. Fernández Bonder and J.D. Rossi. Asymptotic behavior of the best Sobolev trace constant in expanding and contracting domains. Comm. Pure Appl. Anal., 1 (3) (2002), 359-378.

[10] J. Fernández Bonder and J.D. Rossi. On the existence of extremals for the Sobolev trace embedding theorem with critical exponent. Bull. London Math. Soc., 37 (2005), no. 1, 119125.

[11] J. Fernández Bonder, J.D. Rossi and N. Wolanski. Behavior of the best Sobolev trace constant and extremals in domains with holes. Preprint.

[12] J. Fernández Bonder, J.D. Rossi and N. Wolanski. Regularity of the free boundary in an optimization problem related to the best Sobolev trace constant. To appear in SIAM J. Control Optimz.

[13] A. Henrot. Minimization problems for eigenvalues of the Laplacian. J. Evol. Equ. 3(3) (2003), 443-461.

[14] A. Henrot and M. Pierre. Optimization de forme. Book to appear.

[15] T. Kato. Perturbation theory for linear operators. Classics in Mathematics, Springer, Berlin, 1995.

[16] E. Lami Dozo and O. Torne, Symmetry and symmetry breaking for minimizers in the trace inequality. To appear in Comm. Contemp. Math.

[17] Y. Li and M. Zhu. Sharp Sobolev trace inequalities on Riemannian manifolds with boundaries. Comm. Pure Appl. Math., 50 (1997), 449-487.

[18] S. Martinez and J.D. Rossi, Isolation and simplicity for the first eigenvalue of the p-laplacian with a nonlinear boundary condition. Abst. Appl. Anal., 7 (5), (2002), 287-293.

[19] O. Pironeau, Optimal shape design for elliptic systems. Springer, 1984.

[20] L. Schwartz, Cours d'analyse. 1 et 2. Second edition. Hermann, Paris, 1981.

[21] E. Sperner, Spherical symmetrization and eigenvalue estimates. Math. Z., Vol. 176 (1981), 75-86.

[22] M. W. Steklov, Sur les problèmes fondamentaux en physique mathématique, Ann. Sci. Ecole Norm. Sup., Vol. 19 (1902), 455-490. 
JuLián FeRnÁNDEZ BONDER

Departamento de Matemática, FCeyn, Universidad de Buenos Aires,

Pabellón I, Ciudad Universitaria (1428), Buenos Aires, Argentina.

E-mail address: jfbonder@dm.uba.ar

Web page: http://mate.dm.uba.ar/ jfbonder

Pablo Groisman

Instituto de Cálculo, FCEyn, Universidad de Buenos Aires,

Pabellón II, Ciudad Universitaria (1428), Buenos Aires, Argentina.

E-mail address: pgroisma@dm.uba.ar

Web page: http://mate.dm.uba.ar/ pgroisma

Julio D. Rossi

Consejo Superior de Investigaciones Científicas (CSiC), Serrano 117, Madrid, Spain,

on leave from Departamento de Matemática, FCEyN UBA (1428)

Buenos Aires, Argentina.

E-mail address: jrossi@dm.uba.ar

Web page: http://mate.dm.uba.ar/ jrossi 